\title{
Stress Impacts the Regulation Neuropeptides in the Rat Hippocampus and Prefrontal Cortex
}

\author{
Wenxue Li, Aurelie Papilloud, Laura Lozano-Montes, Nan Zhao, Xueting Ye, \\ Xiaozhe Zhang,* Carmen Sandi, and Gregor Rainer
}

\begin{abstract}
Adverse life experiences increase the lifetime risk to several stress-related psychopathologies, such as anxiety or depressive-like symptoms following stress in adulthood. However, the neurochemical modulations triggered by stress have not been fully characterized. Neuropeptides play an important role as signaling molecules that contribute to physiological regulation and have been linked to neurological and psychiatric diseases. However, little is known about the influence of stress on neuropeptide regulation in the brain. Here, we have performed an exploratory study of how neuropeptide expression at adulthood is modulated by experiencing a period of multiple stressful experiences. We have targeted hippocampus and prefrontal cortex (PFC) brain areas, which have previously been shown to be modulated by stressors, employing a targeted liquid chromatography-mass spectrometry (LC-MS) based approach that permits broad peptide coverage with high sensitivity. We found that in the hippocampus, Met-enkephalin, Met-enkephalin-Arg-Phe, and Met-enkephalin-Arg-Gly-Leu were upregulated, while Leu-enkephalin and Little SAAS were downregulated after stress. In the PFC area, Met-enkephalin-Arg-Phe, Met-enkephalin-Arg-Gly-Leu, peptide PHI-27, somatostatin-28 (AA1-12), and Little SAAS were all downregulated. This systematic evaluation of neuropeptide alterations in the hippocampus and PFC suggests that stressors impact neuropeptides and that neuropeptide regulation is brain-area specific. These findings suggest several potential peptide candidates, which warrant further investigations in terms of correlation with depression-associated behaviors.
\end{abstract}

\section{Introduction}

Stress helps individuals to cope with hostile conditions, enhancing their probability of survival. However, continued or repeated exposure to stress can lead to long-lasting negative effects on brain functioning and mental health, such as anxiety and depression. ${ }^{[1,2]}$ For example, convergent evidence suggests that stress during adulthood reduces social behaviors while increases aggression. Importantly, similar effects have been demonstrated when animals experience stress during neurodevelopmental periods, such as for example, peripubertal stress inhibits social interactions during adulthood. Stressors during both developmental periods and adulthood lead to changes in neuronal networks. ${ }^{[3-6]}$ It has thus been shown that peripubertal stress results in modulations of gene and cell adhesion molecule expression in prefrontal cortex (PFC) during adulthood, ${ }^{[4,6]}$ and adulthood stressors also modulate hippocampal functioning. ${ }^{[5]}$ Both hippocampus and PFC are also involved in cognitive and emotional processing of stress stimuli, ${ }^{[7,8]}$ and both hippocampus and PFC mediate the symptoms of depression and anxiety. ${ }^{[9,10]}$ Given that stress can lead to depression and anxiety, we aim to further explore the neurochemical changes on the hippocampus and PFC in adult rats that experienced multiple stressors during both development as well as adulthood. These neurochemical changes are likely to contribute to anxiety and depressive symptoms after stress exposure.

Over the past 30 years, evidence has emerged regarding the role of neuropeptides as signaling molecules in the nervous system. ${ }^{[11-13]}$ Furthermore, it has been suggested that neuropeptide signaling is of special importance for stress responses, as peptides might not be used under normal circumstances, but play a critical role in the nervous system's adaptation to stress. ${ }^{[13-17]}$ Neuropeptides are 50 times larger than neurotransmitters (from 2 to 100 amino acids) and often contain multiple receptor recognition sites. ${ }^{[18]}$ Therefore, they have higher binding affinity (in the order of nmol L-1, while neurotransmitters bind in the order of $\left.\mu \mathrm{mol} \mathrm{L}^{-1}\right) \cdot{ }^{[18]}$ All of these characteristics make neuropeptides highly attractive for drug development, since the 
enhanced selectivity might permit highly selective pharmacological interventions, for example with antagonists, with lower risk for unwanted non-specific side effects. ${ }^{[18]}$ Recently, a substance $P$ receptor (neurokinin 1) antagonist has thus been developed as treatment for major depressive disorder, acting as an efficient serotonin reuptake inhibitor with reduced side effects. ${ }^{[19-21]}$ Additional neuropeptide receptor ligands are currently under clinical trials for several neurological and mental health disorders. ${ }^{[2]}$

Here, we aim to study the effects of a combination of developmental and adulthood stressors on changes on the hippocampal and prefrontal neuropeptides. This study is of specific importance as it could highlight the role of novel therapeutic targets for stress induced neurological disorders (such as anxiety and depression). Previous studies on the effects of stress in neuropeptides have mainly used techniques such as radioimmunoassay or in situ hybridizations for detection of mRNA expression, which are targets for one or a few specific neuropeptides. The use of mass spectrometry (MS) to identify the neuropeptides by contrast allows us to determine the exact sequence (including posttranslational modifications) of all the neuropeptides differentially expressed in our brain region of interest. MS-based neuropeptidomic analysis has successfully resulted in high identification rates of mouse neuropeptides, ${ }^{[23]}$ and we have developed specific techniques allowing broad coverage neuropeptide monitoring in rat and tree shrew animal models. ${ }^{[24,25]}$ Indeed, stress is already known to influence neuropeptide expression in specific brain regions. ${ }^{[3,26-30]}$ As discussed above, a majority of previous studies linking stress with changes on neuropeptides have focused on analyzing changes on one specific neuropeptide.

Given that hundreds of neuropeptides have been identified, ${ }^{[15-18,31,32]}$ it is desirable to obtain a more complete picture of how these signaling molecules are affected by stress, particularly since it is thought that peptides may often act in a concerted, synergistic manner to mediate biological functions. ${ }^{[33]}$ Therefore, identifying the whole range of neuropeptides in the brain region of interest will provide a comprehensive view on the role of neuropeptides on stress responses and will increase the chances of identifying novel pharmacological targets for the treatments of stress-related disorders.

\section{Experimental Section}

\subsection{Animals}

Subjects were the offspring of Wistar Han rats (Charles River Laboratories, France), bred locally in the animal facility. They were kept on a $12 \mathrm{~h}$ light-dark cycle (lights on at $0700 \mathrm{~h}$ ). Food and water were available ad libitum. All procedures were conducted in conformity with the Swiss National Institutional Guidelines on Animal Experimentation and approved by a license from the Swiss Cantonal Veterinary Office Committee for Animal Experimentation.

\subsection{Experimental Design}

Male rats from different litters were distributed into different home cages in groups of two to three non-siblings $21 \mathrm{~d}$ after birth
(P21) and each cage was randomly assigned to control (CTRL, six animals) or peripubertal stress (PPS, six animals) conditions. At P28, animals underwent the well-established peripubertal stress protocol previously developed in Professor Sandi's lab. ${ }^{[34-39]}$ PPS group was exposed to two fear-inducing stressors: the synthetic fox odor trimethylthiazoline (Phero Tech, Delta, BC, Canada) administered in a plastic box $(38 \times 27.5 \times 31 \mathrm{~cm})$ and exposure to an elevated platform $(12 \times 12 \mathrm{~cm})$ presented in a variable schedule in a span of $7 \mathrm{~d}$ across the P28-P42 period. Each stressor lasted for $25 \mathrm{~min}$.

At adulthood, PPS animals followed acute restraint stress procedure for $1 \mathrm{~h}$ before sacrifice while control animals were left undisturbed. Acute restraint stress was induced as follows: rats were gently wrapped in a paper towel to minimize the risk of injuries and restrained $1 \mathrm{~h}$ in wire mesh restrainers $\left(26 \times 26 \mathrm{~cm}^{2}\right)$ in their home cages. Following the sacrifice, brains were rapidly extracted, the hippocampus and PFC parts were dissected within 2 min and immediately denatured with the Denator heat irradiation (Denator AB, Gothenburg, Sweden) as described elsewhere. ${ }^{[40]}$ The denatured tissues were put in the liquid nitrogen rapidly and then transferred to $-80{ }^{\circ} \mathrm{C}$ refrigerator until analysis.

\subsection{Sample Preparation}

The neuropeptides were extracted using a dual phase method for mixing on column (MOC) analysis as introduced in our previous publication. ${ }^{[41]}$ To minimize interference from protein fragmentation, temperature was controlled during all extraction procedures. ${ }^{[42]}$ Each sample was extracted twice, with $0.2 \%$ acetic acid (Fluka, Buchs, Switzerland) aqueous solution and 30\% acetonitrile (Optima grade, Thermo Fisher) containing 0.2\% acetic acid solution. The extraction solution was $5 \mu \mathrm{L}$ per $1 \mathrm{mg}$ brain tissue. A homogenization step was performed on a Precellys 24 homogenizer (Bertin Technologies, Montigny-le-Bretonneux, France) twice for $1 \mathrm{~min}$, and then ultra-sonication for $10 \mathrm{~min}$ at $20^{\circ} \mathrm{C}$. The sample was centrifuged at $20000 \times \mathrm{g}$ for $1 \mathrm{~h}$ at $4{ }^{\circ} \mathrm{C}$. After extraction, the supernatant was filtered on a $10 \mathrm{kDa}$ cutoff filter (Vivacon 500, Sartorious AG, Goettingen, Germany) by centrifuging for approximately $90 \mathrm{~min}$ at $14000 \times \mathrm{g}$ at $4{ }^{\circ} \mathrm{C}$. The dual phase extracts were loaded separately and then mixed on the column for nanoLC-MS analysis. The organic extracts were injected into a trap column first, and after $5 \mathrm{~min}$ the aqueous extracts were also injected such that both extracts were eluted together through the nanoLC-MS system and detected. The injection volume was $10 \mu \mathrm{L}$ and per sample running was almost 90 min.

\subsection{LC-MS Data Acquisition}

The extracts of hippocampus and PFC were analyzed on a LTQDiscovery (Thermo Fisher scientific, Bremen, Germany) tandem with Dionex Ultimate 3000 Nano LC system (Dionex, Thermo Fisher, Sunnyvale, USA). In the LC-MS analysis, the on trap column-mix method was introduced as before. ${ }^{[41]}$ The $2 \mathrm{~cm}$ trap column and $25 \mathrm{~cm}$ analytical column was packed with ReproSilPur C18 AQ particles (100 A, Dr. Maisch GmbH) 5 and $3 \mu \mathrm{m}$, 

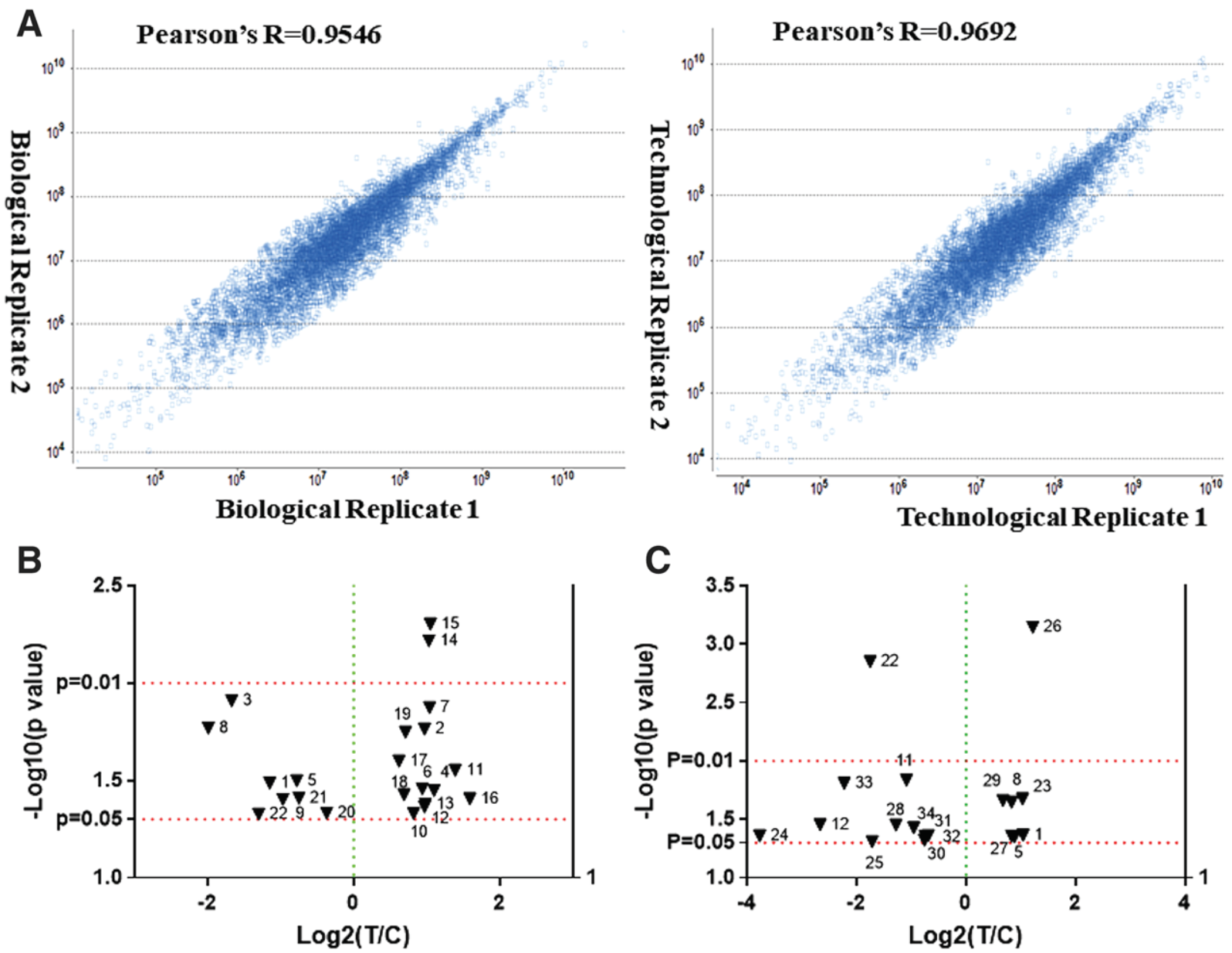

Figure 1. Correlation and volcano plots. A) Biological and technical replicate correlations by ion intensity exported from Progenesis QI for Proteomics program (Nonlinear), which show good reproducibility for label-free neuropeptides quantification; B,C) volcano plots of significantly changed neuropeptides in the hippocampus and PFC, respectively. Numbers used here are linked to those in Figure 2, Figure 3, and Table 1. There are 22 and 18 significant changed neuropeptides detected in hippocampus and PFC brain regions in peripubertal stress animals compared with controls.

respectively. The empty analytical capillary (75 $\mu \mathrm{m}$ ID, $360 \mu \mathrm{m}$ OD, with $8 \mu \mathrm{m}$ emitter tip) was purchased from New Objective (Cambridge, USA). The loading pump was always washed with $98 \%$ water and $1.98 \%$ acetonitrile containing $0.2 \%$ formic acid. The loading status was set to 3 min and then switched to injection position until $80 \mathrm{~min}$. The NC pump mobile phase consisted of water:acetonitrile:formic acid of 98:1.98:0.2 and water:acetonitrile:formic acid of 1.98:98:0.2 for A and B, respectively. The elution was performed at flow rate $300 \mathrm{~nL} \mathrm{~min}^{-1}$ with $90 \mathrm{~min}$ gradient profile as follows: $0-3 \mathrm{~min}, 5 \% \mathrm{~B}$; 3-7 $\mathrm{min}, 5-$ $20 \%$ B; 7-35 min, 20-30\% B; 30-55 min, 30-45\% B; 55-60 min, 45-55\% B; 60-65 min, 55-90\% B; 65-80 min, 90\% B, and the last $10 \mathrm{~min}$ for equilibration with $5 \% \mathrm{~B}$.

\subsection{Differential Analysis and Targeted Analysis for Peptide Identification}

For the quantification analysis, all the hippocampus $(n=6$ for each animal group) and PFC ( $n=6$ for each animal group) samples were arranged alternating treatment samples and controls, and each sample was analyzed in two replicates. For improving quantification accuracy, quality control samples (QCs) derived froma mixture of treatments and controls (each $20 \mu \mathrm{L}$ ), were used (four QCs for hippocampus and PFC, at the beginning and end of the sequence). For the differential analysis, the full scan was acquired from $350-2000 \mathrm{~m} / z$ with Orbitrap analyzer with resolve power 30000 . The lock mass (445.120025 from polydimethylcyclosiloxane) was used for real-time internal recalibration. ${ }^{[43]}$ In differential analysis, the Progenesis for proteomics software (Nonlinear Dynamic, Newcastle, UK) was used for the ion intensity extraction for treatments, controls, and QCs and exported as CSV files. The unpaired $t$-test between treatment and control group was performed, and the level of statistical significance was set at $p<0.05(n=6)$. Significantly, peptide ions were rejected if the coefficient variation was greater than $50 \%$ for QCs $(n=4)$. After filter, the ions were exported as CSV file and imported into the inclusion list with 3 min of the retention window for further targeted LC-MS/MS analysis, by which the peptides were selected for 
collision-induced dissociation (CID) fragmentation. Acquisition on LC-MS instrument consisted of a full MS scan event and three MS/MS scan events at mass range from 350-2000 m/z, and the setting of resolve power 30000 for MS and MS/MS acquisition. Minimum signal threshold was set as 100 000, and isolation window was $2 \mathrm{~m} / z$. Normalized collision energy was $30 \%$. For the dynamic exclusion setting, the repeat count of 2 , exclusion, and repeat duration time of $30 \mathrm{~s}, 50 \mathrm{ppm}$ tolerance for precursor selection with disabled preview mode for full MS scan and disabled monoisotopic precursor selection mode.

\subsection{Data Analysis}

The PEAKS Studio 5.3 (BSI, Waterloo, Ontario, Canada), which has Data Refinement, Auto De Novo, and Peaks Search functions, was used for the spectral interpretation of all raw MS/MS data files. ${ }^{[4,45]}$ The mass tolerance of precursor ions and products were specified to $10 \mathrm{ppm}$ and $0.05 \mathrm{Da}$, respectively. No enzyme was selected for the database search. Variable posttranslational modifications, including amidation (C-terminal), acetylation (N-terminal), pyroglutamylation from glutamic acid, and glutamine (N-terminal) were selected for de novo sequencing. The Uniprot rat database (January 4, 2016) was specified for the database search after de novo sequencing. The cutoff of false discovery rate for peptide identification in Peaks Search was 1\%.
After that, the manual check was used for inspecting identified neuropeptides. ${ }^{[41]}$

\section{Results}

The purpose of this study was to profile the alteration of neuropeptides in rat hippocampus and PFC in animals subjected to multiple stressors compared to control animals (not subjected to stress). We estimated the technical and biological replicates, taking into account both instrumentrelated and biological variability, that showed good reproducibility in peptides quantitative analysis (Figure 1A). In summary, hippocampus neuropeptide differential analysis results revealed 22 significant changed neuropeptides (non-paired $t$-test, $p<0.05$, see Figure $1 \mathrm{~B}$ ) derived from four precursors secretogranin-1, proenkephalin-A (PENK), cerebellin-1, and ProSAAS. Of these modulated neuropeptides, 14 of them were upregulated and eight were downregulated in the stress treatment compared with controls (Figure 2 and Table 1). In PFC, 18 neuropeptides (non-paired t-test, $p<0.05$, see Figure 1B) exhibited stress-related modulations. These peptides were derived from seven precursors: secretogranin-1, PENK, cholecystokinin (CCK), vasoactive intestinal peptide (VIP), somatostatin, ProSAAS, and Pro-melanin-concentrating hormone (Pro-MCH). Of these neuropeptides, seven were upregulated

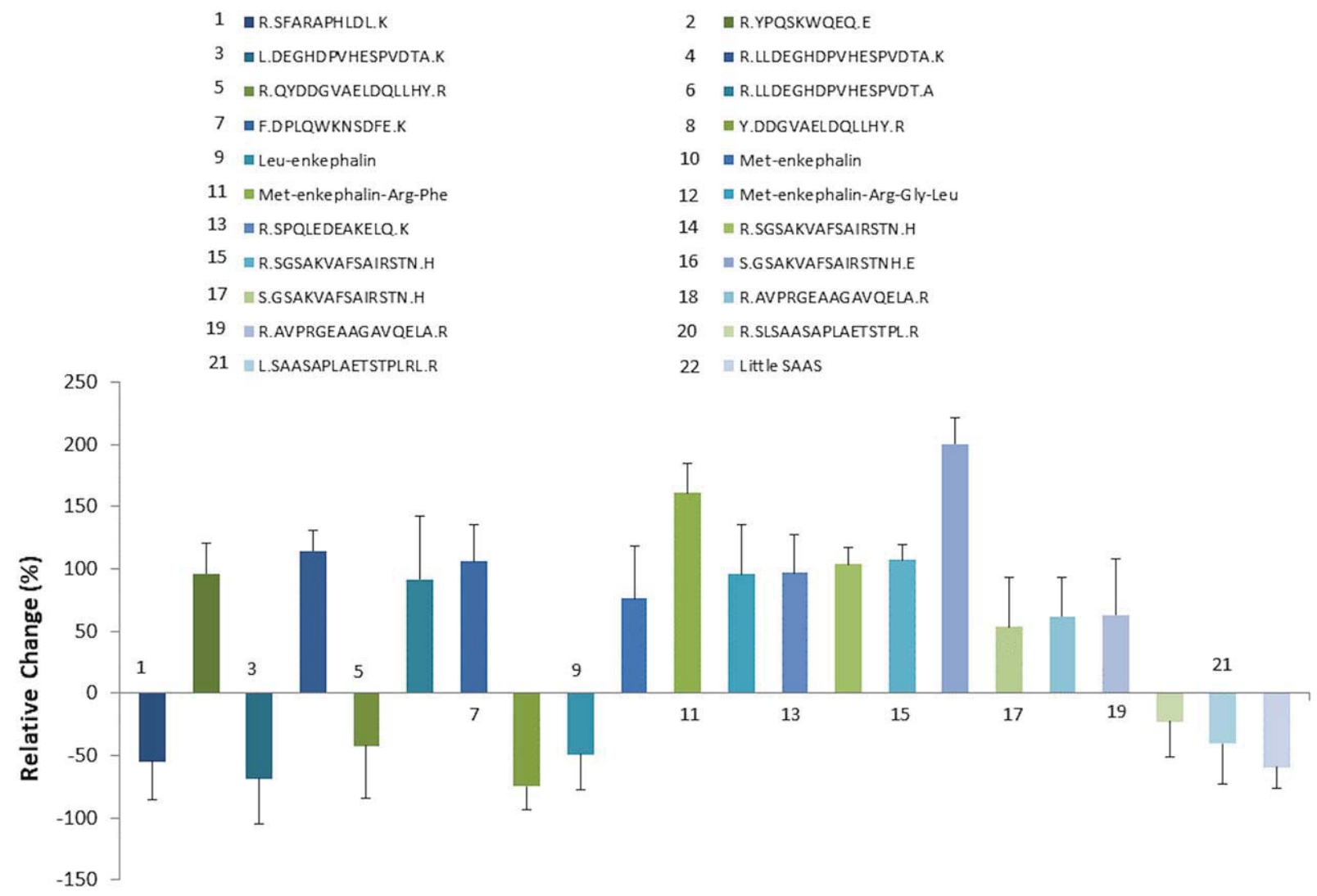

Figure 2. Histogram for significantly changed neuropeptides detected in the hippocampus after peripubertal stress treatment (T) compared with controls (C). The labeled numbers are linked to those in Figure 1B volcano plots and Table 1. The complete list of neuropeptide information is shown in Supporting Information Table 1. 
Table 1. Significantly changed neuropeptides in the hippocampus and PFC brain regions in peripubertal stress treatment animals compared with controls.

\begin{tabular}{|c|c|c|c|c|c|}
\hline & No. & Precursor & Peptide & Name & $p$-value \\
\hline \multirow[t]{22}{*}{ Hippocampus } & 1 & Secretogranin- 1 & R.SFARAPHLDL.K & & 0.033 \\
\hline & 2 & Secretogranin- 1 & R.YPQSKWQEQ.E & & 0.017 \\
\hline & 3 & Secretogranin- 1 & L.DEGHDPVHESPVDTA.K & & 0.012 \\
\hline & 4 & Secretogranin- 1 & R.LLDEGHDPVHESPVDTA.K & & 0.036 \\
\hline & 5 & Secretogranin- 1 & R.QYDDGVAELDQLLHY.R & & 0.032 \\
\hline & 6 & Secretogranin-1 & R.LLDEGHDPVHESPVDT.A & & 0.035 \\
\hline & 7 & Secretogranin-1 & F.DPLQWKNSDFE.K & & 0.013 \\
\hline & 8 & Secretogranin- 1 & Y.DDGVAELDQLLHY.R & & 0.017 \\
\hline & 9 & Proenkephalin-A & R.YGGFL.K & Leu-enkephalin & 0.04 \\
\hline & 10 & Proenkephalin-A & K.YGGFM.K & Met-enkephalin & 0.047 \\
\hline & 11 & Proenkephalin-A & R.YGGFMRF & Met-enkephalin-Arg-Phe & 0.028 \\
\hline & 12 & Proenkephalin-A & R.YGGFMRGL.K & Met-enkephalin-Arg-Gly-Leu & 0.043 \\
\hline & 13 & Proenkephalin-A & R.SPQLEDEAKELQ.K & & 0.042 \\
\hline & 14 & Cerebellin-1 & R.SGSAKVAFSAIRSTNH.E & & 0.006 \\
\hline & 15 & Cerebellin-1 & R.SGSAKVAFSAIRSTN.H & & 0.005 \\
\hline & 16 & Cerebellin-1 & S.GSAKVAFSAIRSTNH.E & & 0.039 \\
\hline & 17 & Cerebellin-1 & S.GSAKVAFSAIRSTN.H & & 0.025 \\
\hline & 18 & ProSAAS & S.APLAETSTPLRL.R & & 0.037 \\
\hline & 19 & ProSAAS & R.AVPRGEAAGAVQELA.R & & 0.018 \\
\hline & 20 & ProSAAS & R.SLSAASAPLAETSTPL.R & & 0.047 \\
\hline & 21 & ProSAAS & L.SAASAPLAETSTPLRL.R & & 0.039 \\
\hline & 22 & ProSAAS & R.SLSAASAPLAETSTPLRL.R & Little SAAS & 0.047 \\
\hline \multirow[t]{18}{*}{ Prefrontal cortex } & 1 & Secretogranin- 1 & R.SFARAPHLDL.K & & 0.043 \\
\hline & 5 & Secretogranin-1 & R.QYDDGVAELDQLLHY.R & & 0.045 \\
\hline & 8 & Secretogranin- 1 & Y.DDGVAELDQLLHY.R & & 0.023 \\
\hline & 23 & Secretogranin-1 & R.Q(-17.03)YDDGVAELDQLLHY.R & & 0.021 \\
\hline & 24 & Secretogranin-1 & R.LGALFNPYFDPLQWKNSDFE.K & & 0.044 \\
\hline & 25 & Proenkephalin-A & R.VGRPEWWMDYQ.K & & 0.049 \\
\hline & 11 & Proenkephalin-A & R.YGGFMRF & Met-enkephalin-Arg-Phe & 0.015 \\
\hline & 12 & Proenkephalin-A & R.YGGFMRGL.K & Met-enkephalin-Arg-Gly-Leu & 0.035 \\
\hline & 26 & Cholecystokinin & R.AVLRPDSEPRARLGALLA.R & & 0.001 \\
\hline & 27 & Cholecystokinin & R.AVLRPDSEPRARLGALL.A & & 0.044 \\
\hline & 28 & Cholecystokinin & R.MSVLKNLQGLDPSHRISD.R & & 0.035 \\
\hline & 29 & Cholecystokinin & V.LRPDSEPRARLGALL.A & & 0.022 \\
\hline & 30 & Cholecystokinin & R.LGALLARYIQQVR.K & & 0.043 \\
\hline & 31 & Cholecystokinin & K.NLQGLDPSHRISD.R & & 0.044 \\
\hline & 32 & Vasoactive intestinal peptide & R.HADGVFTSDYSRLLGQISAKKYLESLI(-.98).G & Instestinal peptide $\mathrm{PHI}-27$ & 0.048 \\
\hline & 33 & Somatostatin & R.SANSNPAMAPRE.R & Somatostatin-28(AA1-12) & 0.016 \\
\hline & 22 & ProSAAS & R.SLSAASAPLAETSTPLRL.R & Little SAAS & 0.001 \\
\hline & 34 & Pro-MCH & E.IGDEENSAKFPI (-.98).G & & 0.037 \\
\hline
\end{tabular}

The neuropeptide numbers are linked to those in Figure 1 volcano plots and histograms in Figures 2 and 3. The complete neuropeptide information is shown in Supporting Information Table 1

and 11 downregulated following the stressors (see Figure 3 and Table 1). The representative MS/MS spectrums for neuropeptides were shown at Figure 4. The complete list of identified and quantified neuropeptides are shown in Supporting Information, Tables 1 and 2.

The largest group of neuropeptides affected by stress in the hippocampus were derived from the secretogranin-1 precursor $(n=8)$. Four of these were downregulated including
R.SFARAPHLDL.K $(-55 \%, p=0.033)$, L.DEGHDPVHESPVDTA.K $(-69 \%, p=0.012)$, R.QYDDGVAELDQLLHY.R $(-42 \%$, $p=0.032)$, and Y.DDGVAELDQLLHY.R $(-75 \%, p=0.017)$, while another four were upregulated including R.YPQSKWQEQ.E (96\%, $p=0.017)$, R.LLDEGHDPVHESPVDTA.K (115\%, $p=0.036)$, R.LLDEGHDPVHESPVDT.A ( $92 \%, p=0.035)$, and F.DPLQWKNSDFE.K $(106 \%, p=0.013)$. In PFC neuropeptides, five neuropeptides derived from secretogranin-1 precursor 

1 R.SFARAPHLDL.K
8 @Y.DDGVAELDQLLHY.R
$24=$ R.LGALFNPYFDPLQWKNSDFE.K
11 Met-enkephalin-Arg-Phe
26 M.AVLRPDSEPRARLGALLA.R
$28 \approx$ R.MSVLKNLQGLDPSHRISD.R
$30=$ R.LGALLARYIQQVR.K
32 =1 Instestinal peptide $\mathrm{PHI}-27$
$22=$ Little SAAS

5 @ R.QYDDGVAELDQLLHY.R

$23=R . Q(-17.03) Y D D G V A E L D Q L L H Y . R$

25 aR.VGRPEWWMDYQ.K

2 met-enkephalin-Arg-Gly-Leu

27 R.AVLRPDSEPRARLGALL.A

29 V.LRPDSEPRARLGALL.A

$31=$ K.NLQGLDPSHRISD.R

$33=$ Somatostatin-28

$34=$ Pro-MCH

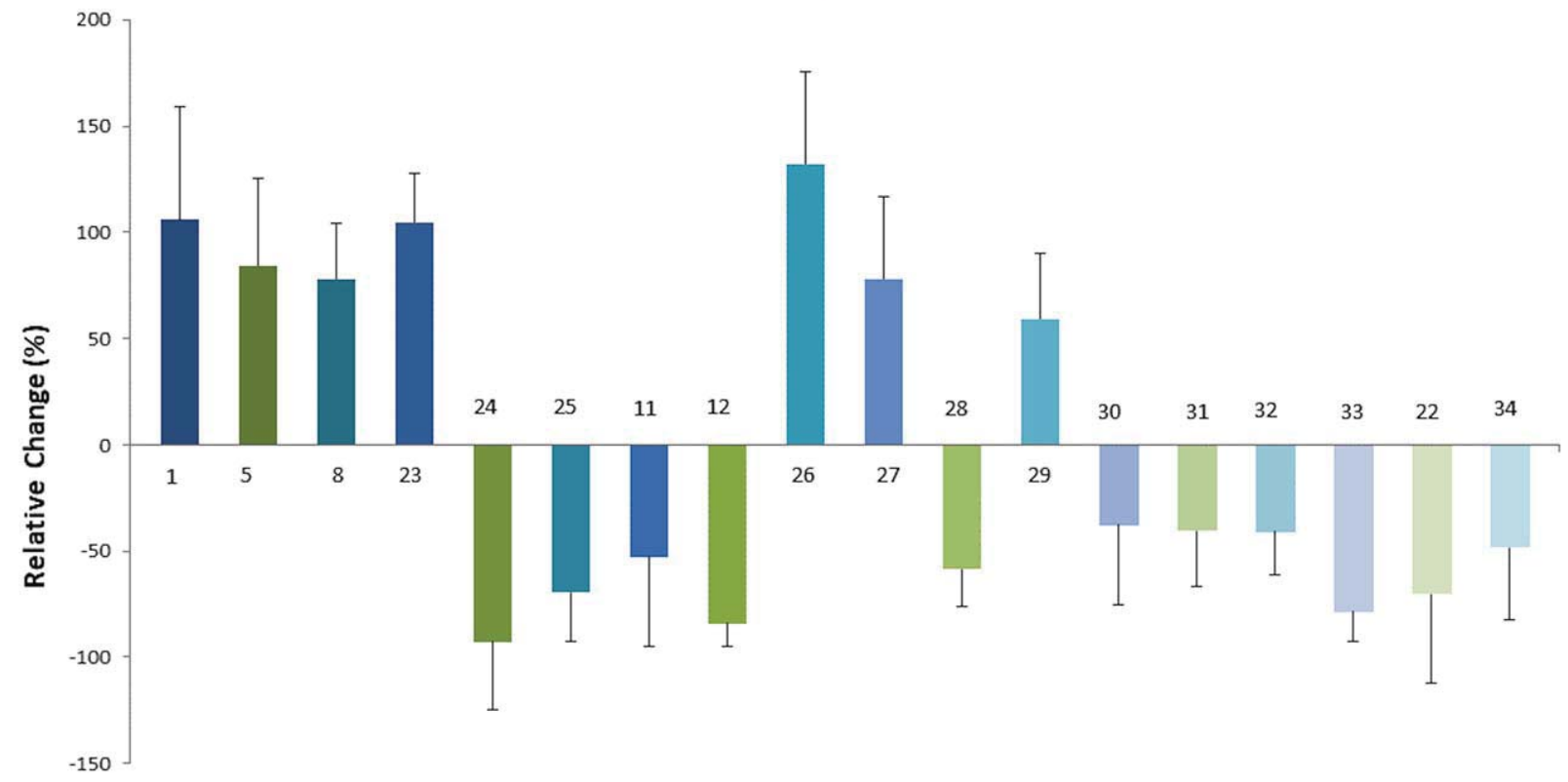

Figure 3. The histogram for significant changed neuropeptides detected in PFC after peripubertal stress treatment ( $T$ ) compared with controls (C). The labeled numbers are linked to those in Figure 1C volcano plot and Table 1. The complete list of neuropeptide information is shown in Supporting Information Table 1.

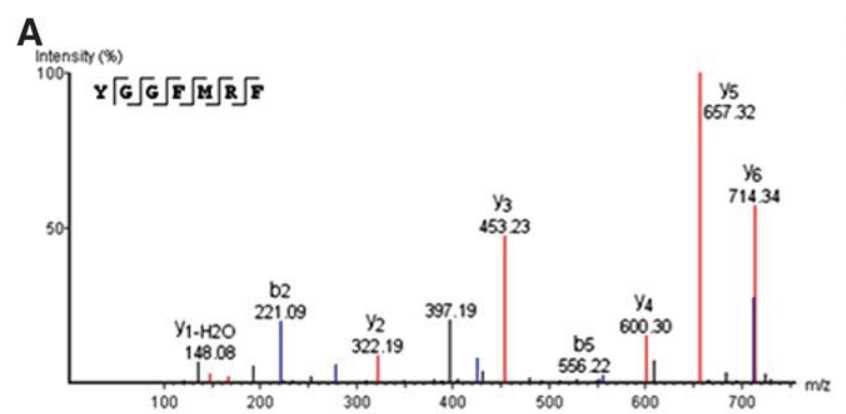

\section{B}

C

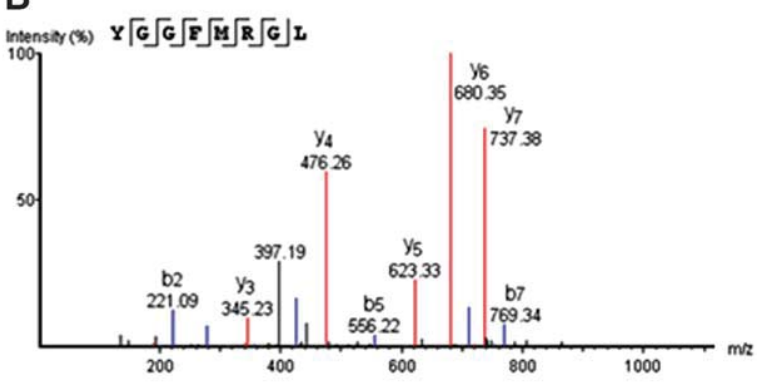

intensity (\%)
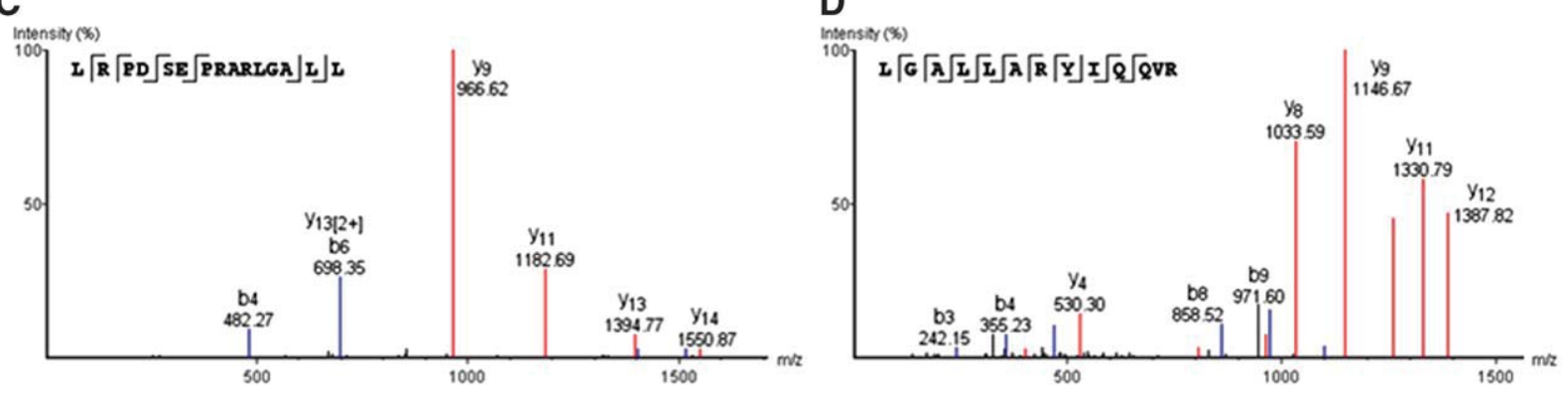

Figure 4. The representative MS/MS spectrums for neuropeptides identification with PEAKs studio. A, Met-enkephalin-Arg-Phe; B, Met-enkephalin-ArgGly-Leu; C and D neuropeptides cleaved from precursor Cholecystokinin (CCK). 
were affected by stress, and four of these being upregulated including R.SFARAPHLDL.K (106\%, $p=0.043)$, R.QYDDGVAELDQLLHY.R (84\%, $p=0.045)$, Y.DDGVAELDQLLHY.R (78\%, $p=0.023)$, and R.Q(-17.03) YDDGVAELDQLLHY $(105 \%, p=0.021)$, while only the single peptide R.LGALFNPYFDPLQWKNSDFE.K was downregulated $-92 \%(p=0.044)$. It has been reported that peptides derived from secretogranin-1 precursor: SEESQEREY, SFARAPQLD, LLDEGHDPVHESPIDTA, and LLDEGHYPV were downregulated in mouse hypothalamus after antidepressant imipramine treatment. ${ }^{[46]}$ Note that the rat peptide R.LLDEGHDPVHESPVDTA.K we detected here is highly similar to the previously documented mouse peptide LLDEGHDPVHESPIDTA, having only one amino acid difference that probably reflects organism variation. The second largest group of stress-modulated neuropeptides in the hippocampus was derived from the PENK precursor with four of them upregulated including Met-enkephalin (77\%, $p=0.047)$, Met-enkephalin-Arg-Phe (161\%, $p=0.028)$, Met-enkephalinArg-Gly-Leu (96\%, $p=0.043)$, R.SPQLEDEAKELQ.K (97\%, $p=0.042$ ), and peptide Leu-enkephalin downregulated $-49 \%$ $(p=0.04)$. Of the peptide family, three altered peptides were detected in the PFC and all of these were downregulated: R.VGRPEWWMDYQ.K, Met-enkephalin-Arg-Phe, and Metenkephalin-Arg-Gly-Leu at $-69 \%(p=0.049),-52 \%(p=0.015)$, and $-84 \%(p=0.035)$, respectively. Additional modulated peptides belonged to diverse families. In the hippocampus, four peptides derived from cerebellin-1 precursor were observed, all of which were upregulated: R.SGSAKVAFSAIRSTNH.E $(104 \%, p=0.006)$, R.SGSAKVAFSAIRSTN.H (107\%, $p=0.005)$, S.GSAKVAFSAIRSTNH.E $(200 \%, p=0.039)$, and S.GSAKVAFSAIRSTN.H $(54 \%, p=0.025)$. Previous mouse hypothalamic peptides research has revealed decreased levels of SGSAKVAFSAIRSTNH, SGSAKVAFSAIRSTN, and GSAKVAFSAIRSTN after imipramine treatment. ${ }^{[46]}$ We document for the first time an involvement of five ProSAAS precursor derived peptides in the brain stress response. Peptides R.SLSAASAPLAETSTPL.R, L.SAASAPLAETSTPLRL.R, and R.SLSAASAPLAETSTPLRL.R (Little SAAS) were downregulated $-23 \%(p=0.047),-40 \%(p=0.039)$, and $-59 \%(p=0.047)$, respectively. S.APLAETSTPLRL.R and R.AVPRGEAAGAVQELA.R were upregulated $62 \%(p=0.037)$ and $63 \%(p=0.018)$, respectively. In the PFC, we also observed six altered peptides derived from the CCK precursor and the levels of three of them were upregulated. Peptides R.AVLRPDSEPRARLGALLA.R, R.AVLRPDSEPRARLGALL.A, and V.LRPDSEPRARLGALL.A were upregulated $132 \%$ ( $p=0.001), 78 \%(p=0.044)$, and $59 \%$ $(p=0.022)$, respectively, and R.MSVLKNLQGLDPSHRISD.R, R.LGALLARYIQQVR.K, and K.NLQGLDPSHRIS D.R were downregulated $-59 \%(p=0.035),-38 \%(p=0.043)$ and $-41 \%(p=0.044)$, respectively. This observation is consistent with previous results implicating CCK neurotransmission in anxiogenic and depressogenic effects of social stress. ${ }^{[28,47,48]}$ We found that intestinal peptide PHI-27 (R.HADGVFTSDYSRLLGQISAKKYLESLI(-.98).G), derived from VIP precursor, was downregulated $-41 \%(p=0.048)$ in the PFC. VIP has been implicated in modulating emotional processes or adaptive responses to stressful stimuli in rat model of depression. ${ }^{[49]}$ Somatostatin-28 (AA1-12) was downregulated
$-78 \%(p=0.016)$ in brain PFC following stress. The Little SAAS (R.SLSAASAPLAETSTPLRL.R) peptide derived from ProSAAS was also downregulated $-70 \%(p=0.001)$ in PFC. In addition to this, peptide E.IGDEENSAKFPI(-.98).G derived from Pro-MCH precursor was also downregulated $-48 \%(p=0.037)$ in the PFC region. It has been reported that the neuropeptide $\mathrm{MCH}$ plays a role in rapid eye movement (REM) sleep and is involved in the pathophysiology of depression. ${ }^{[50]}$ Some authors have demonstrated that $\mathrm{MCH}$ receptor-1 antagonist (SNAP-7941) possesses antidepressant and anxiolytic effects in animal models of depressive disorder. ${ }^{[51-54]}$ In addition, repeated chronic mild stress could increase the levels of gene expression of $\mathrm{MCH}$ receptor- 1 in mice hippocampus and this was reversed by chronic fluoxetine treatment. ${ }^{[55]}$ Besides, the importance of MCHergic system in depressive disorder was emphasized in a recent study, indicating that an upregulation on the level of preproMCH expression and a decrease of $\mathrm{MCH}$ receptor could be a biomarker of the severity of depressive disorders. ${ }^{[56]}$ These results suggest that MCHergic system, precursors, or produced neuropeptides are related to certain aspects of major depressive disorder.

\section{Discussion}

The peptidomics approach for the differential analysis has an exclusive advantage compared with other methods, which allows simultaneous detection and identification of a large number of peptides and provides the ability to differentiate between unique peptides derived from the same precursor. It is important to note that in neuropeptide analysis, where there is no enzyme digestion, all of the detected peptide ions are of endogenous origin and may thus contribute to a better understanding of the underlying neurobiological processes. Developing novel pharmacological strategies to treat psychiatric disorders is thus an important current direction in neurobiology, and a large body of evidence suggests that neuropeptides may play a critical role in these diseases. ${ }^{[57]}$

The hypothalamic-pituitary-adrenal (HPA) axis participates in the stress response and plays a key role in maintenance of homeostasis. ${ }^{[58]}$ Moreover, HPA dysregulation has been linked to depression. ${ }^{[59]}$ The hippocampus and PFC are highly susceptible to stress. ${ }^{[60,61]}$ A decreased metabolism in the PFC is a frequently replicated finding in major depressive disorder. ${ }^{[61,62]}$ Here, we use quantified differential analysis for hippocampus and PFC neuropeptide detection and quantification in the rat after multiple stressors. In total, we observed only a limited number of neuropeptides impacted by stress, some of which were consistent with previous reports whereas others were demonstrated here for the first time to be linked to stress. A group of neuropeptides derived from secretogranin-1 (LLDEGHYPVRESPIDTA), cerebellin-1 (SGSAKVAFSAIRSTNH), and somatostatin precursors were downregulated in the mouse hypothalamus after antidepressant imipramine acute or chronic treatment ${ }^{466}$; accordingly, in the present study we found upregulation of all of these peptides in hippocampus and PFC after stressors. Therefore, our results were consistent with previous literature, given that stressors can trigger depression, it is expected that antidepressant treatment regulates neuropeptides in the opposite way that 
stressors do. This is a very relevant result given that it highlights a potential neurobiological mechanism by which stressors could lead to depression. Furthermore, it shows the consistency of the secretogranin-1, cerebellin-1, and somatostatin precursor regulation between different brain regions: hypothalamus, PFC, and hippocampus in different animal species (mice and rats, respectively).

The opioid peptide enkephalin is an important modulator of stress responses, ${ }^{[63]}$ and our findings support this, as members of its family were strongly modulated by stressor application. It has been reported that enkephalin knockout male mice appear to be resistant to chronic mild stress, ${ }^{[29]}$ and PENK knockout mice exhibit abnormal stress reactivity and increased anxiety behavior in acute stress situations. ${ }^{[64,65]}$ In wild-type mice, PENK gene expression levels are increased in the PFC, hippocampus, and other brain areas, but decreased in the amygdala following chronic mild stress. ${ }^{[29]}$ Furthermore, chronic mild stress significantly decreases levels of Met-enkephalin in the rat nucleus accumbens. ${ }^{[66]}$ It has been previously demonstrated that key enkephalin-derived neuropeptides Leu-enkephalin, Metenkephalin, Met-enkephalin-Arg-Phe, and Met-enkephalin-ArgGly-Leu were upregulated by chronic nicotine treatment in rat dorsal striatum. ${ }^{[41]}$ Many of these peptides were also affected by stress in the present study, partially in a similar, and partially in opposite directions as in the chronic nicotine study. This indicates substantial overlap in neuropeptide regulatory mechanisms between nicotine consumption and stress, which is interesting given that both of these experiences can trigger depressive episodes in humans.

Related to enkephalins, the present results highlight two novel aspects of neuropeptide regulation. First, it shows that members of the same neuropeptide family can be both up- and downregulated in a given brain area following stressors. This emphasizes the power of MS-based approaches, in that we can provide the exact amino acid sequence of each modulated peptide. Our finding reinforces the idea that neuropeptides are coregulated, but that this regulation is complex and probably involves multiple excitatory and inhibitory interactions among compounds of the same peptide family. Second, we show clearly that peptide expression in different brain areas following stressor is not regulated in the same way, but rather there are specific aspects that may be characteristic of how each brain structure in the network responds to stress. For example, we see that while in the hippocampus the majority of enkephalin-derived peptides are upregulated, all of the ones detected in the PFC were downregulated. This observation suggests that the synthesis and release of neuropeptides is not brain-wide, but specific to brain regions. Our results have important implications for the designing of peptides as potential drug targets; major functional brain regions should be studied independently avoiding the assumption that neuropeptide regulation follows the same patterns over the whole brain.

To our knowledge, alterations of intestinal peptide PHI-27 (VIP) in the PFC following stress are reported for the first time in the present study. It is however known that hippocampal VIP-positive neurons contribute to modulating emotional processes or adaptive responses to stressful stimuli in a rat model of depression. ${ }^{[49,67]}$ Decades ago, it was also reported that VIP decreases in the cerebrospinal fluid in atypical depression. ${ }^{[68]}$ VIP enhances both pre- and postsynaptic GABA-ergic transmission in hippocampal interneurons, leading to increased excitatory synaptic transmission to CA1 pyramidal cells, ${ }^{[69]}$ a mechanism that modulates neuronal excitability in the rat hippocampus. ${ }^{[0]}$ Finally, we also observed neuropeptides derived from the precursor CCK in this study, consistent with an involvement of CCKergic neurotransmission in both anxiogenic and depressogenic effects of social stress. ${ }^{[28]}$ CCK thus contributes to PFC regulation of depression and anxiety-like behaviors, and CCK receptor blockade in medial PFC induces a resilient phenotype, whereas CCK administration into medial PFC mimics the anxiogenic- and depressogenic-like effects of social stress. ${ }^{[47]}$ Available evidence thus supports a role of CCK in the regulation of depression and anxiety, but the mechanism needs to be further clarified.

\section{Conclusion}

This is the first study that uses the MS-based quantitative method to profile alterations in a broad range of neuropeptides in the rat hippocampus and PFC after stressor treatment. This method is able to high throughput detect and quantify peptides including their corresponding post-transnational modifications, which is not achieved by others biochemical methods currently. With the label free relative quantification strategy, we found several significant changes in classic neuropeptides in hippocampus and PFC, including Met-enkephalin, somatostatin, VIP, and corresponding derivative peptides. Most of these peptides exhibit consistent regulation with previous described stress animal models. These results reveal that the brain responses to stressful episodes are multifaceted and involves brain region specific modulations of peptide regulation. Our findings provide a novel and comprehensive approach to evaluate neurochemical circuits in animal models related to stress or other related disorders, for example, depression. The detected peptides can now be examined in terms of their links with behavioral symptoms and underlying neural circuits, paving the way for potential biomarker discovery or new pharmacological strategies for treatment of stress-related disorders in the future.

\begin{abstract}
Abbreviations
AA, amino acid; CCK, cholecystokinin; CID, collision induced dissociation; HPA, hypothalamic-pituitary-adrenal; LC-MS, liquid chromatography-mass spectrometry; LTQ, linear trap quadrupole; MOC, mix on column; MS, mass spectrometry; PENK, proenkephalin; $\mathrm{PFC}$, prefrontal cortex; $\mathrm{PHI}$, porcine intestinal peptide; Pro- $\mathrm{MCH}$, Pro-melanin-concentration hormone; QC, quality control; REM, rapid eye movements; VIP, vasoactive intestinal peptide
\end{abstract}

\section{Supporting Information}

Supporting Information is available 


\section{Acknowledgements}

This work is supported by the grants MOST 2015DFG424460, NSF 21475128, and CAS 100 Talent Project.

\section{Conflict of Interest}

The authors declare no competing financial interest.

\section{Keywords}

adulthood stress, anxiety, depression, differential analysis, hippocampus, neuropeptides, peripuberty stress, prefrontal cortex

[1] K. S. Kendler, L. M. Karkowski, C. A. Prescott, Am. J. Psychiatry 1999, 156,837

[2] S. E. Smith, J. Li, K. Garbett, K. Mirnics, P. H. Patterson, J. Neurosci.: Off. J. Soc. Neurosci. 2007, 27, 10695.

[3] A. H. Veenema, A. Blume, D. Niederle, B. Buwalda, I. D. Neumann, Eur. J. Neurosci. 2006, 24, 1711

[4] J. Márquez-Ruiz, R. Leal-Campanario, R. Sánchez-Campusano, B. Molaee-Ardekani, F. Wendling, P. C. Miranda, G. Ruffini, A. Gruart, J. M. Delgado-García, Proc. Natl. Acad. Sci. 2012, 109, 6710.

[5] M. A. van der Kooij, M. Fantin, I. Kraev, I. Korshunova, J. Grosse, O. Zanoletti, R. Guirado, C. Garcia-Mompo, J. Nacher, M. G. Stewart, V. Berezin, C. Sandi, Neuropsychopharmacol.: Off. Publ. Am. Coll. Neuropsychopharmacol. 2014, 39, 1148.

[6] S. Bariselli, S. Tzanoulinou, C. Glangetas, C. Prevost-Solie, L. Pucci, J. Viguie, P. Bezzi, E. C. O'Connor, F. Georges, C. Luscher, C. Bellone, Nat Neurosci. 2016, 19, 926.

[7] J. P. Herman, H. Figueiredo, N. K. Mueller, Y. Ulrich-Lai, M. M. Ostrander, D. C. Choi, W. E. Cullinan, Front. Neuroendocrinol. 2003, 24, 151.

[8] G. E. Tafet, R. Bernardini, Progr. Neuro-Psychopharmacol. Biol. Psychiatry 2003, 27, 893.

[9] V. Krishnan, E. J. Nestler, Nature 2008, 455, 894.

[10] P. A. Keedwell, C. Andrew, S. C. Williams, M. J. Brammer, M. L. Phillips, Biol. Psychiatry 2005, 58, 843.

[11] P. C. Montecucchi, R. de Castiglione, S. Piani, L. Gozzini, V. Erspamer, Int. J. Pept. Protein Res. 1981, 17, 275.

[12] M. G. Rosenfeld, J. J. Mermod, S. G. Amara, L. W. Swanson, P. E. Sawchenko, J. Rivier, W. W. Vale, R. M. Evans, Nature 1983, 304, 129.

[13] T. Hokfelt, T. Bartfai, F. Bloom, Lancet. Neurol. 2003, 2, 463.

[14] C. De la Rosa-Prieto, D. Saiz-Sanchez, I. Ubeda-Banon, A. FloresCuadrado, A. Martinez-Marcos, Front. Neurosci. 2016, 10, 227.

[15] J. R. Schank, A. E. Ryabinin, W. J. Giardino, R. Ciccocioppo, M. Heilig, Neuron 2012, 76, 192.

[16] G. A. Carrasco, L. D. Van de Kar, Eur. J. Pharmacol. 2003, 463, 235.

[17] F. Reichmann, P. Holzer, Neuropeptides 2016, 55, 99.

[18] T. Hökfelt, T. Bartfai, F. Bloom, Lancet Neurol. 2003, 2, 463.

[19] M. S. Kramer, N. Cutler, J. Feighner, R. Shrivastava, J. Carman, J. J. Sramek, S. A. Reines, G. Liu, D. Snavely, E. Wyatt-Knowles, J. J. Hale, S. G. Mills, M. MacCoss, C. J. Swain, T. Harrison, R. G. Hill, F. Hefti, E. M. Scolnick, M. A. Cascieri, G. G. Chicchi, S. Sadowski, A. R. Williams, L. Hewson, D. Smith, E. J. Carlson, R. J. Hargreaves, N. M. Rupniak, Sci. (N. Y.) 1998, 281, 1640

[20] A. N. Martinez, M. T. Philipp, J. Neurol. Neuromed. 2016, 1, 29.

[21] M. Kramer, Eur. Psychiatry 2002, 17, 10
[22] G. Griebel, F. Holsboer, Nat. Rev. Drug Disc. 2012, 11, 462.

[23] X. Zhang, F. Petruzziello, F. Zani, L. Fouillen, P. E. Andren, G. Solinas, G. Rainer, J. Proteome Res. 2012, 11, 2819.

[24] F. Petruzziello, L. Fouillen, H. Wadensten, R. Kretz, P. E. Andren, G. Rainer, X. Zhang, J. Proteome Res. 2012, 11, 886.

[25] L. Fouillen, F. Petruzziello, J. Veit, A. Bhattacharyya, R. Kretz, G. Rainer, X. Zhang, J. Proteomics 2013, 80, 311.

[26] I. I. Rybkin, Y. Zhou, J. Volaufova, G. N. Smagin, D. H. Ryan, R. B. Harris, Am. J. Physiol.-Regulat. Integrative Comparative Physiol. 1997, 273, R1612.

[27] C. Becker, M. Hamon, J.-J. Benoliel, Neuropharmacology 1999, 38, 525.

[28] C. Becker, M. H. Thiebot, Y. Touitou, M. Hamon, F. Cesselin, J. J. Benoliel, J. Neurosci. 2001, 21, 262.

[29] I. Melo, E. Drews, A. Zimmer, A. Bilkei-Gorzo, Genes Brain Behav. 2014, 13, 550.

[30] A. Thorsell, P. Svensson, L. Wiklund, W. Sommer, R. Ekman, M. Heilig, Regul. Pept. 1998, 75, 247.

[31] A. F. Russo, Headache: J. Head Face Pain 2017, 57, 37.

[32] I. Prieto, A. Segarra, M. de Gasparo, M. Ramírez-Sánchez, AIMS NEUROSCI. 2016, 3, 487.

[33] D. Chen, K. P. Taylor, Q. Hall, J. M. Kaplan, Genetics 2016, 204, 1151.

[34] C. Márquez, G. L. Poirier, M. I. Cordero, M. H. Larsen, A. Groner, J. Marquis, P. J. Magistretti, D. Trono, C. Sandi, Translat. Psychiatry 2013, 3, e216.

[35] C. Kohl, X.-D. Wang, J. Grosse, C. Fournier, D. Harbich, S. Westerholz, J.-T. Li, A. Bacq, C. Sippel, F. Hausch, Psychoneuroendocrinology 2015, $55,128$.

[36] V. Veenit, O. Riccio, C. Sandi, J. Psychiatr. Res. 2014, 53, 1.

[37] S. Tzanoulinou, C. García-Mompó, E. Castillo-Gómez, V. Veenit, J. Nacher, C. Sandi, PLoS One 2014, 9, e94666.

[38] G. L. Poirier, N. Imamura, O. Zanoletti, C. Sandi, J. Psychiatr. Res. 2014, 57, 157

[39] M. I. Cordero, F. Ansermet, C. Sandi, Psychoneuroendocrinology 2013, $38,2758$.

[40] M. Svensson, M. Boren, K. Skold, M. Falth, B. Sjogren, M. Andersson, P. Svenningsson, P. E. Andren, J. Proteome Res. 2009, 8, 974.

[41] F. Petruzziello, S. Falasca, P. E. Andren, G. Rainer, X. Zhang, Mol. Cell. Proteomics: MCP 2013, 12, 1553.

[42] M. Svensson, K. Skold, A. Nilsson, M. Falth, K. Nydahl, P. Svenningsson, P. E. Andren, Anal. Chem. 2007, 79, 15, 18.

[43] J. V. Olsen, L. M. de Godoy, G. Li, B. Macek, P. Mortensen, R. Pesch, A. Makarov, O. Lange, S. Horning, M. Mann, Mol. Cell. Proteomics: MCP 2005, 4, 2010.

[44] B. Ma, K. Zhang, C. Hendrie, C. Liang, M. Li, A. Doherty-Kirby, G. Lajoie, Rapid Commun. Mass Spectrom. 2003, 17, 2337.

[45] Y. Han, B. Ma, K. Zhang, J. Bioinform. Comput. Biol. 2005, 3, 697.

[46] A. Nilsson, N. Stroth, X. Zhang, H. Qi, M. Falth, K. Skold, D. Hoyer, P. E. Andren, P. Svenningsson, Neuropharmacology 2012, 62, 347.

[47] V. Vialou, R. C. Bagot, M. E. Cahill, D. Ferguson, A. J. Robison, D. M. Dietz, B. Fallon, M. Mazei-Robison, S. M. Ku, E. Harrigan, C. A. Winstanley, T. Joshi, J. Feng, O. Berton, E. J. Nestler, J. Neurosci. 2014, $34,3878$.

[48] C. Becker, B. Zeau, C. Rivat, A. Blugeot, M. Hamon, J. J. Benoliel, Mol. Psychiatry 2008, 13, 1079.

[49] M. Ivanova, S. Belcheva, I. Belcheva, Z. Stoyanov, R. Tashev, Acta Neurobiol. Exp. (Warsz) 2014, 74, 317.

[50] P. Torterolo, C. Scorza, P. Lagos, J. Urbanavicius, L. Benedetto, C. Pascovich, X. Lopez-Hill, M. H. Chase, J. M. Monti, Front Neurosci. 2015, 9, 475 .

[51] B. Borowsky, M. M. Durkin, K. Ogozalek, M. R. Marzabadi, J. DeLeon, B. Lagu, R. Heurich, H. Lichtblau, Z. Shaposhnik, I. Daniewska, T. P. Blackburn, T. A. Branchek, C. Gerald, P. J. Vaysse, C. Forray, Nat. Med. 2002, 8, 825 . 
[52] S. Chung, G. S. Parks, C. Lee, O. Civelli, J. Mol. Neurosci.: MN 2011 43, 115.

[53] D. J. David, K. C. Klemenhagen, K. A. Holick, M. D. Saxe, I. Mendez, L. Santarelli, D. A. Craig, H. Zhong, C. J. Swanson, L. G. Hegde, X. I. Ping, D. Dong, M. R. Marzabadi, C. P. Gerald, R. Hen, J. Pharmacol. Exp. Ther. 2007, 321, 237.

[54] T. Shimazaki, T. Yoshimizu, S. Chaki, CNS Drugs 2006, 20, 801.

[55] M. Roy, N. David, M. Cueva, M. Giorgetti, Biol. Psychiatry 2007, 61, 174.

[56] M. J. Garcia-Fuster, G. S. Parks, S. M. Clinton, S. J. Watson, H. Akil, O. Civelli, Eur. Neuropsychopharmacol.: J. Eur. Coll. Neuropsychopharmacol. 2012, 22, 607.

[57] V. Kormos, B. Gaszner, Neuropeptides 2013, 47, 401.

[58] Y. M. Ulrich-Lai, J. P. Herman, Nat. Rev. Neurosci. 2009, 10, 397.

[59] G. P. Chrousos, Nat. Rev. Endocrinol. 2009, 5, 374.

[60] M. Pandya, M. Altinay, D. A. Malone Jr., A. Anand, Curr. Psychiatry Rep. 2012, 14, 634.

[61] S. Rigucci, G. Serafini, M. Pompili, G. D. Kotzalidis, R. Tatarelli, World J. Biol. Psychiatry 2010, 11, 165.
[62] T. A. Kimbrell, T. A. Ketter, M. S. George, J. T. Little, B. E. Benson, M. W. Willis, P. Herscovitch, R. M. Post, Biol. Psychiatry 2002, 51, 237.

[63] P. Berube, J.-F. Poulin, S. Laforest, G. Drolet, Neuropsychopharmacology 2014, 39, 1159.

[64] J. C. Kung, T. C. Chen, B. C. Shyu, S. Hsiao, A. C. Huang, J. Biomed. Sci. 2010, 17, 29.

[65] A. Ragnauth, A. Schuller, M. Morgan, J. Chan, S. Ogawa, J. Pintar, R. J. Bodnar, D. W. Pfaff, PNAS 2001, 98, 1958.

[66] M. Dziedzicka-Wasylewska, M. Papp, Pol. J. Pharmacol. 1996, $48,53$.

[67] I. Belcheva, M. Ivanova, R. Tashev, S. Belcheva, Peptides 2009, 30, 1497.

[68] A. Gjerris, O. J. Rafaelsen, P. Vendsborg, J. Fahrenkrug, J. F. Rehfeld, J. Affect. Disord. 1984, 7, 325.

[69] D. Cunha-Reis, A. M. Sebastiao, K. Wirkner, P. Illes, J. A. Ribeiro, Br. J. Pharmacol. 2004, 143, 733.

[70] H. L. Haas, B. H. Gahwiler, Neuroscience 1992, 47, 273. 